\title{
A Promising Therapeutic Approach for Treatment of Posterior Uveitis: Recombinant T Cell Receptor Ligand Protects Lewis Rats from Acute and Recurrent Experimental Autoimmune Uveitis
}

\author{
Grazyna Adamus $^{a} \quad$ Landon J. Karren $^{a} \quad$ Jeff Mooney ${ }^{b}$ Gregory G. Burrows ${ }^{b, c}$ \\ ${ }^{a}$ Ocular Immunology Laboratory, Department of Ophthalmology, and Departments of ${ }^{\text {b Neurology and }}$ \\ 'Biochemistry and Molecular Biology, Oregon Health and Science University, Portland, Oreg., USA
}

\section{Key Words}

Experimental autoimmune uveitis, rat $\cdot \mathrm{RTL}$, treatment $\cdot$ Cytokine $\cdot$ Chemokine

\begin{abstract}
Introduction: Chronic autoimmune uveitis is a major cause of vision loss from intraocular inflammation in humans. In this study we report that a recombinant TCR ligand (RTL220) composed of the $\alpha 1$ and $\beta 1$ domains of MHC class II molecules linked to the uveitogenic interphotoreceptor retinoidbinding protein (IRBP) 1177-1191 peptide is effective in the suppression of acute and recurrent experimental autoimmune uveitis (EAU). Material and Methods: EAU was induced with IRBP1177-1191 peptide or by adoptive transfer of specific $T$ cells in Lewis rats. The rats received 5 doses of RTL220 subcutaneously every other day starting at the onset of clinic signs of EAU. Results: The administration of RTL220 resulted in a delayed onset and a significant amelioration of the disease severity at clinical levels and showed protection of the retina from inflammatory damage at histological levels. In treatment of recurrent EAU, RTL220 administrated at the first or second onset of clinical disease significantly inhibited EAU, modulated immune responses and provided protection from relapses of uveitis. The systemic and local proinflammatory cytokines were significantly re-
\end{abstract}

duced, including IL-17. There was local and systemic increase in IL-10 and reduction in the expression of the proinflammatory chemokines CCL2, CCL3 and CCL5. Conclusions: Our studies demonstrate a successful treatment of acute and recurrent EAU with RTL220, which effectively suppressed the recurrence of inflammation and reversed clinical and histological EAU by altering cytokine and chemokine expression. These findings strongly support a possible clinical application of this novel class of peptide/MHC class II drugs for patients with autoimmune uveitis.

Copyright $\odot 2010$ S. Karger AG, Basel

\section{Introduction}

Uveitis, a sight-threatening inflammatory disease of the eye, can result from immune-mediated responses against ocular antigens. Recurrent ocular inflammation, which occurs in $\sim 50 \%$ of the uveitis cases, is the major morbidity because following a resolution of an acute episode, the patient's vision is frequently decreased by chronic subclinical inflammatory reactions that may eventually lead to blindness [1]. The conventional treatment includes corticosteroids and immunosuppressive agents, which are limited by their side effects and only efficient in approximately half of the recurrent ocular inflamma-

\section{KARGER}

두 2010 S. Karger AG, Basel

Fax +41613061234 E-Mail karger@karger.ch www.karger.com www.karger.com/ore
Grazyna Adamus

Casey Eye Institute-BRB, Mail code L467AD, Oregon Health and Science University

3181 SW Sam Jackson Park Rd

Portland, OR 97239 (USA)

Tel. +1 503418 2540, Fax +1 503418 2541, E-Mail adamusg@ ohsu.edu 
tion patients $[2,3]$. Animal models of experimental autoimmune uveitis (EAU) have been useful in testing new therapeutic approaches to treat intraocular inflammation [4].

For uveitis to develop, autoreactive T cells must be activated outside the eye and then pass the blood-ocular barrier, enter the eye and crossreact with ocular autoantigens. In autoimmune uveitis, Th1 and Th17 cells play an important role in the pathogenicity of the disease [5]. IRBP is the major uveitogenic retinal antigen eliciting EAU $[4,6]$. In immunologically normal mice or rats, EAU is a T-cell-mediated disease that targets the photoreceptor cells where the target antigens are located, which often leads to an irreversible destruction of photoreceptor cells and subsequently to the loss of vision [4,7]. Mice and rats genetically predisposed to a predominantly Th1 or Th17 response differ in the severity of EAU that they develop [5].

MHC class I and class II are molecules that influence the immunological susceptibility of many types of noninfectious uveitis [8]. Vogt-Koyanagi-Harada (VKH) disease, sympathetic ophthalmia and birdshot retinochoroidopathy are conditions that show a significant MHC association with both MHC class II molecules and MHC class I molecules, indicating an autoimmune pathogenesis for uveitis [9]. Over the years, a number of different approaches to treatments have been investigated $[2,10$, 11]. Blocking the antigen presentation function of $\mathrm{MHC}$ class II molecules by competitor peptides has been proposed as a possible therapeutic approach for MHC-associated autoimmune diseases [12-14]. Recombinant TCR ligands (RTLs) consisting of covalently linked $\beta 1$ and $\alpha 1$ domains of MHC class II molecules that are tethered to specific antigenic peptides represent minimal TCR ligands and are a new class of drugs treating autoimmune inflammation $[15,16]$. These compounds are developed to bind selectively to the specific TCR of interest and were shown to effectively prevent and reverse clinical and histological signs of experimental autoimmune encephalomyelitis (EAE) in an antigen-specific manner $[17,18]$.

We have previously demonstrated that RTL201 bearing the nonretinal antigen, myelin basic protein peptide $\mathrm{MBP}_{72-89}$, completely suppressed autoimmune anterior uveitis associated with EAE in Lewis rats [19]. In the present study, our goal was to design a new RTL bearing the retina-specific antigenic peptide IRBP1177-1191 peptide (RTL220) and determine its efficacy in the treatment of acute and, in particular, recurrent EAU (R-EAU). T cells responsive to IRBP and arrestin (S-antigen) have been found in the blood of patients with uveitis, which suggests that these proteins may be the major autoantigens involved in human uveitis $[4,20,21]$. Our findings show a RTL220 can successfully treat R-EAU by altering cytokine and chemokine expression. These results support a possible clinical application in the therapy of autoimmune uveitis.

\section{Material and Methods}

\section{Animals}

Female Lewis rats (Harlan Sprague-Dawley, Inc., Indianapolis, Ind., USA), 6-8 weeks old, were used in these studies. The rats were housed at the Oregon Health and Science University Animal Care Facility according to institutional and federal guidelines. All animal experimentation procedures adhered to the ARVO Resolution on the Use of Animals in Research and have been approved by the institutional animal committee. The experimental groups consisted of 5 or 6 rats and the experiments were repeated at least twice.

Generation and Production of RTL220

The major epitope of IRBP is composed of amino acid residues 1177-1191 (IRBP1177-1191; also called R16 [22, 14]) and was used to design and express our new RTL220 gene. The RTL220 construct is comprised of a single gene encoding a polypeptide of 220 amino acids containing the $\beta 1$ and $\alpha 1$ domains of the rat MHC class II (RT1.B) and the covalently tethered peptide IRBP11771191 (ADGSSWEGVGVVPDV) (fig. 1). The plasmid sequences were confirmed as described previously [15]. The construct was expressed in Escherichia coli. RTL220 was purified by chromatography following the same methodology that was used to make the 'empty' (RTL101; $\beta 1 \alpha 1$ chain only) and antigen-coupled RTL constructs described previously [15]. RTL203 bearing the covalently tethered cardiac myosin peptide CM-2 was used as an irrelevant control [19].

\section{Induction and Assessment of EAU}

Acute EAU. Lewis rats were immunized with $100 \mu \mathrm{l}$ of $20 \mu \mathrm{g}$ IRBP1177-1191 in complete Freund's adjuvant supplemented with $2 \mathrm{mg} / \mathrm{ml}$ of Mycobacterium tuberculosis. The rats were evaluated for clinical signs of EAU daily by biomicroscopy. The intensity of inflammation was scored blind on an arbitrary scale of 0-4 as follows: 0 - no disease, 1 - engorged blood vessels in the iris and abnormal pupil configuration, 2 - hazy anterior chamber, 3 - moderate opaque anterior chamber with the pupil visible and 4 - opaque anterior chamber, obscure pupil and proptosis.

Recurrent EAU. R-EAU was induced by adoptive transfer of IRBP1177-1191-specific T cells as described recently [23]. Donor Lewis rats were initially immunized with $20 \mu \mathrm{g}$ of IRBP1177-1191 in complete Freund's adjuvant and M. tuberculosis. Ten days later, their spleens were removed, and the suspension of splenocytes was stimulated with $20 \mu \mathrm{g} / \mathrm{ml}$ IRBP1177-1191 peptide for $48 \mathrm{~h}$. Blasts were collected and injected intraperitoneally at $5 \times 10^{6} \%$ dose per recipient rat. The rats were examined daily for clinical signs of inflammation by biomicroscopy for about 39 days following the $\mathrm{T}$ cell transfer. 
Treatment with RTLs

RTLs at $300 \mu \mathrm{g}$ per dose were injected subcutaneously in the back at the time of IRBP immunization or at the onset of clinical EAU signs. The rats received 5 doses of RTL220 every other day. To treat R-EAU, 5 dosages of $300 \mu \mathrm{g}$ of RTL220 were administrated starting at the first or second onset of clinical EAU followed by weekly injections of $300 \mu \mathrm{g}$ of RTL220 subcutaneously for the duration of the experiment.

\section{Histology}

The eyes were removed and fixed in formalin and then processed for paraffin embedding. Tissue sections $(5 \mu \mathrm{m})$ were stained with hematoxylin and eosin for histopathological scoring. EAU was scored on a scale of 0 (no disease) to 4 (maximum disease), based on the presence of inflammatory cell infiltration of the iris, cilliary body, anterior chamber and retina, as follows: $0=$ normal anterior and retinal architecture, no inflammatory cells in these structures; 1 = mild inflammatory cell infiltration of anterior segment and retina; 2 = moderate inflammatory cell infiltration of anterior segment and retina; $3=$ massive inflammatory cell infiltration of anterior segment and retina, disorganized anterior segment and retina, and $4=$ massive inflammatory cell infiltration of anterior segment and retina, disorganized anterior segment and retina, and photoreceptor cell damage [24].

\section{Cytokine/Chemokine Analysis}

The eyes were collected at the end of the experiment. Cytokines and chemokines were extracted from each eye with PBS by homogenization [25]. The soluble fraction was collected by centrifugation from 2 individual eyes of different rats from 2 experiments (IL-13 in 3 eyes) and then used as a cytokine source for determination. The aliquots from each eye were tested in duplicate. Splenocytes obtained from individual spleens were cultured at $5 \times 10^{5}$ cells/well in a 96 -well flat-bottom culture plate in RPMI stimulation medium with $20 \mu \mathrm{g} / \mathrm{ml}$ IRBP peptide for $48 \mathrm{~h}$. Supernatants were harvested and stored at $-80^{\circ} \mathrm{C}$ until testing for $\mathrm{cy}$ tokines in triplicate. A customized rat Beadlyte multiplex cytokine detection kit (Millipore) was used to detect the following cytokines and chemokines: IFN- $\gamma$, IL-1 $\beta$, IL-4, IL-6, IL-17, TNF$\alpha$, IL-10, IL-2, IL-13, MCP-1, MIP- $1 \alpha$ and RANTES simultaneously as described by the manufacturer's protocol. The signals were analyzed on a Luminex 200 (Bio-Rad), and the data were analyzed using Bio-Plex Management software (Bio-Rad).

Statistical Analyses

Statistical analysis was performed with Prism Graph Pad software using a 2-tailed Student $t$ test or the significance between the controls and treatment groups was determined by 1-way analysis of variance (ANOVA). Differences with a p value $<0.05$ were considered significant and are denoted by an asterisk.

\section{Results}

\section{RTL220 Inhibits IRBP1177-1191-Induced EAU}

The studies were designed to determine the efficacy of a new RTL220 bearing the retina-specific antigenic IRBP1177-1191 peptide in the treatment of acute and, in

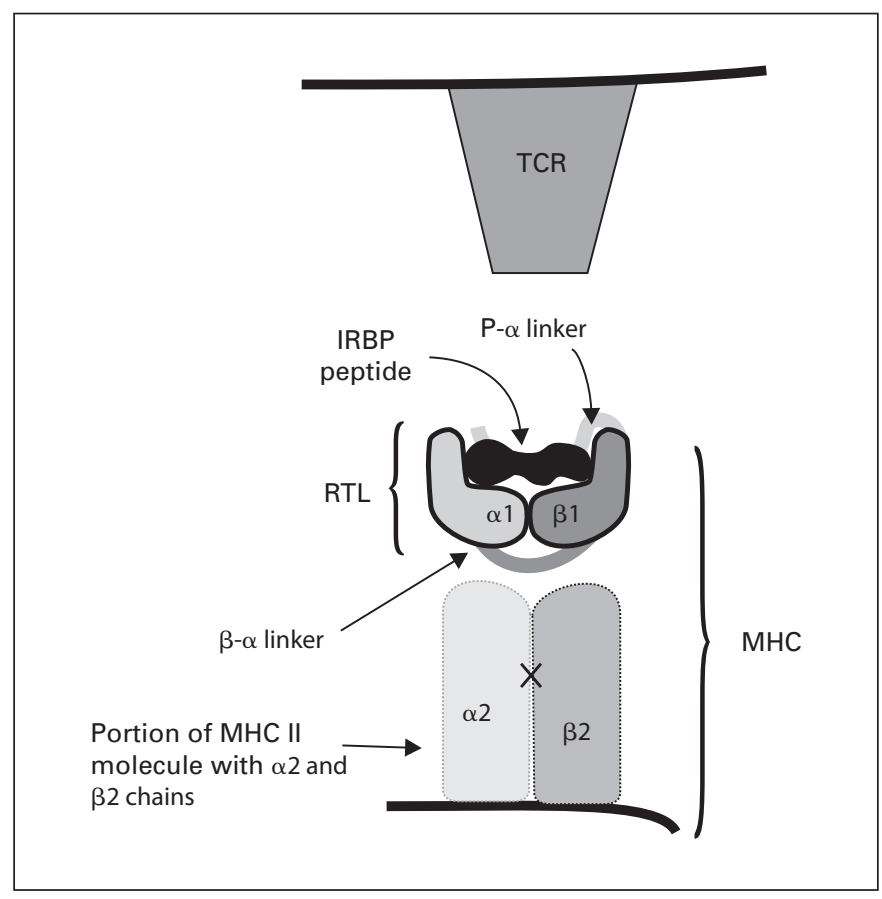

Fig. 1. RTL220 drug used in the study: RTL is composed of the $\alpha 1$ and $\beta 1$ domains of MHC class II molecules linked to the uveitogenic IRBP1177-1191 peptide.

particular, recurrent EAU. The therapeutic effects of RTL220 were evaluated in Lewis rats with EAU induced with IRBP1177-1191. Inflammatory infiltrates are found in both anterior and posterior parts of the eye. Therefore, we were able to identify clinical signs using biomicroscopy. Inflammatory changes in the posterior part and retinal damage were assessed by histology. The rats uniformly developed eye inflammation, which occurred on days 9-10 following the antigen injection. Typically, the inflammation diminished after 25 days.

Two treatment regimens consisting of 5 doses of 300 $\mu \mathrm{g}$ RTL220 administered every 2 days were tested in the following groups: (1) treatment started on day 1 , concurrent with immunization of the IRBP antigen, and (2) treatment started at the onset of clinical inflammatory signs as assessed by biomicroscopy. The controls included: untreated rats, treated with empty RTL101 without peptide and RTL203 carrying the irrelevant cardiac myosin peptide CM-2. Figure 2 shows collective results from 1 typical experiment. RTL220 effectively reduced the clinical signs of EAU ( $p<0.0004$ for all groups, $\mathrm{n}=6$; 1-way ANOVA). In the treatment regimens, we observed delayed onset by $3-4$ days and a considerable ame- 


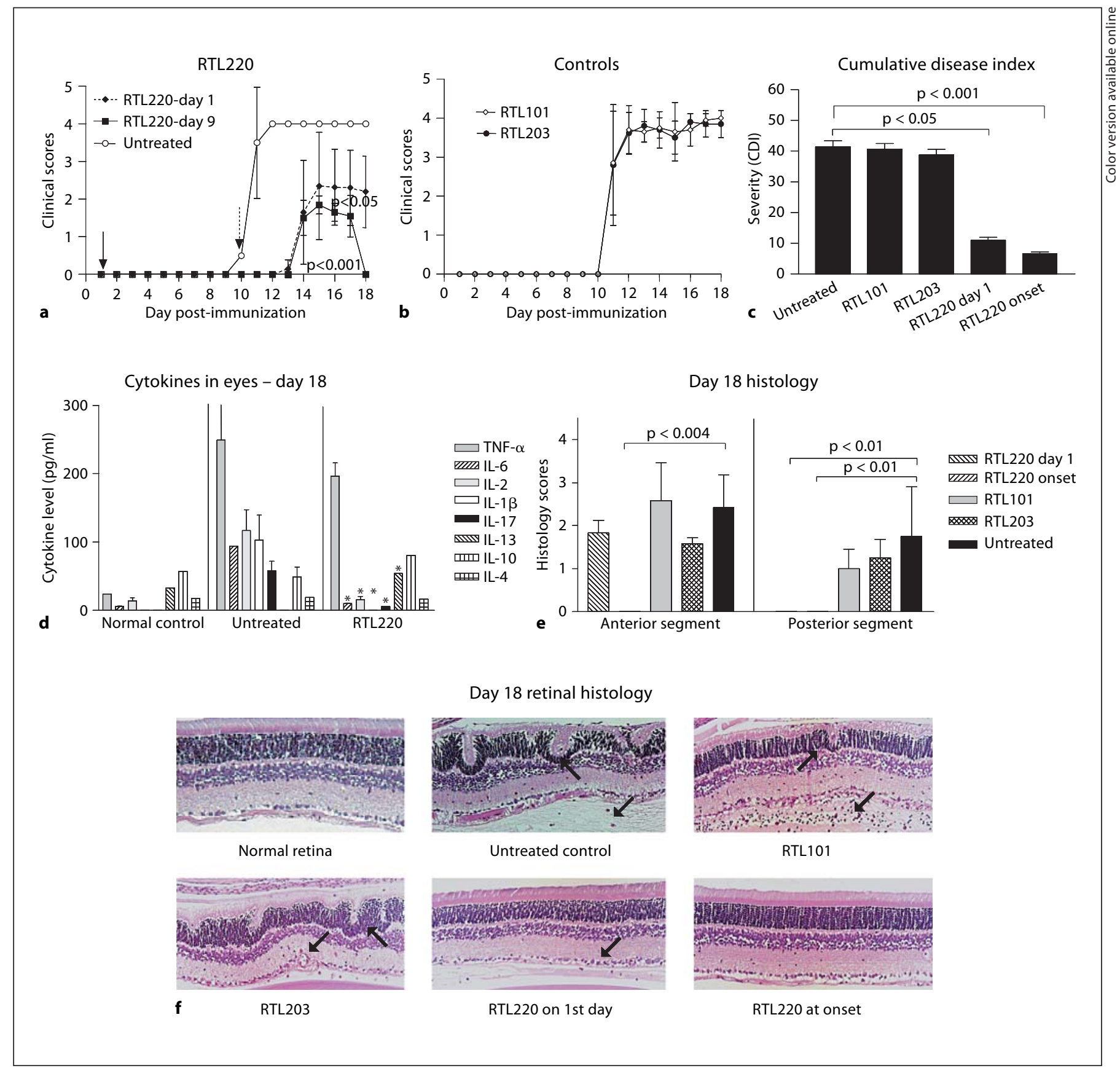

Fig. 2. Inhibition of IRBP1177-1191-induced EAU with RTL220 in Lewis rats. Time course of EAU in RTL-treated rats with 5 doses of $300 \mu \mathrm{g}$ of RTL220, RTL101 or RTL203 subcutaneously starting on the day of IRBP immunization (a) or at the onset of clinical signs (b). The data present an average of 10 eyes for 5 rats and SD from a representative experiment. The severity of inflammation was determined as described in the methods. The significance between the controls and treatment groups was determined by 1 -way ANOVA, overall $p=0.0004$. c Overall severity of EAU. Bars indicate CDIs calculated for each group $(n=5)$ in this experiment. d Level of cytokines in individual eyes from unimmunized rats (control), immunized with IRBP peptide (untreated) and not treated, and RTL220-treated rats (RTL220) from rats treated at the onset of clinical signs ( $\mathrm{n}=4$ eyes). Beadlyte multiplex cytokine detection kit (Millipore) was used to measure cytokines as described in the methods. The significance between the control and treatment group was determined using Student's t test $\left({ }^{*} \mathrm{p}<0.05\right)$ and is denoted by an asterisk. e Summary of histology scores for each group. Bars represent average histology scores for anterior and posterior segments of all eyes $(n=10)$ in experimental groups. f Representative histology of the retina from different treatment groups as shown in a and $\mathbf{b}$. Arrows indicate inflammatory cells and retinal waves. 
lioration of disease severity and duration compared to the control groups (fig. 2a, b). The rats that were treated starting at disease onset showed complete suppression of EAU after 5 days of mild uveitis (fig. 2b). Nonspecific RTL203 and empty RTL101 did not alter the course of EAU, demonstrating the specificity of the RTL treatment. The histological results from acute EAU agreed with clinical assessments. The control eyes (untreated, RTL203 and 'empty' RTL101) showed the presence of inflammatory cells in the anterior chamber, vitreous and iris and infiltrating the retina. Positive clinical scores in some treated EAU rats were consistent with the presence of some lingering inflammatory cells in the anterior portion of the eye. The posterior segment was mostly free of infiltrating cells (day 18). RTL220 treatment of active EAU, administered at disease onset, essentially eliminated the infiltration of inflammatory cells into the eye and fully preserved the retina. RTL220 treatment that started on day 1 with the disease induction was less efficient in preventing eye inflammation than treatment starting at onset (fig. 2e, f) and markedly lowered the severity of inflammation in the retina to $30 \%$, compared to untreated control rats. Although diminished mild anterior inflammation was detected in the anterior chamber during the course of disease, the posterior segment was clear of inflammatory cells.

To compare the overall severity of disease in the course of the illness we calculated the cumulative disease index (CDI) and the results are presented for both eyes of each rat in experimental and control groups in figure $2 \mathrm{a}$ and $\mathrm{b}$ (fig. 2c). The CDIs indicated that RTL220 significantly inhibited EAU in rats treated at the time of immunization $(p<0.05)$ as well as at the onset of clinical EAU $(p<0.01)$ compared to untreated controls. Pro- and anti-inflammatory cytokines were examined in the eyes of rats treated with RTL220 at the onset of clinical EAU and IBRPinjected untreated rat eyes at the end of the experiment on day 18 after immunization. Figure $2 \mathrm{~d}$ shows the significant reduction in the proinflammatory cytokine levels of IL-2, IL-1 $\beta$ and IL-17 ( $p<0.05$ denoted by an asterisk) in RTL-treated eyes as compared to untreated rats. TNF- $\alpha$, INF- $\gamma$ (not shown) and IL- 6 change was reduced but did not reach statistical significance. Anti-inflammatory IL-13 was increased, IL-10 was slightly increased, and IL- 4 was unchanged in treated and untreated eyes.

\section{RTL220 Suppresses Recurrence of}

IRBP1177-1191-Induced EAU

We wished to examine whether RTL220 can also affect the course of recurrence of EAU. To study these effects, we used the Lewis rat model of recurrent uveitis induced by adoptive transfer of $5 \times 10^{6}$ IRBP1177-1191-specific T cells. R-EAU is characterized by early disease onset on day 3 after T cell transfer in all rats and by 3 relapses over 40 days that varied in individual rats. We compared untreated rats to rats treated with 5 doses of $300 \mu \mathrm{g}$ injected every 2 days, followed by $300 \mu \mathrm{g}$ of RTL220 once a week for the duration of the experiment. Regimen 1 started at the onset of the first attack of R-EAU and regimen 2 at the second attack of EAU. Because the clinical course varied, which is consistent with the previously published data [23], we show representative results for each eye of 2 out of 6 rats per group from both treatment regimens and untreated controls in figure 3 . The regimen starting at the first onset of clinical signs drastically reduced the severity of EAU and stopped the recurrences of EAU in all treated rats

Fig. 3. Treatment of EAU relapses with RTL220 induced with $\mathrm{T}$ cell transfer in Lewis rats at the first onset of clinical disease. a, b Clinical course for 2 control rats - clinical scores represent each eye for 2 untreated controls. c, d Two RTL220-treated rats - the clinical time course is presented for 2 eyes of individual rats treated at the onset of clinical disease with 5 doses of $300 \mu \mathrm{g}$ of RTL subcutaneously every other day followed by weekly 1-dosage treatments. e, f Treatment of EAU relapses with RTL220 at the second onset of clinical disease in Lewis rats. The rats were given 6 doses of $300 \mu \mathrm{g}$ of RTL220 every other day followed by weekly 1 -dosage treatments. The significance between controls and treatment groups was determined by 1-way ANOVA, overall $\mathrm{p}<$ 0.0001 . Arrows indicate the time of RTL220 administration. g Level of local and systemic cytokines in R-EAU in RTL220treated rats. Individual eyes and spleens were collected at the end of the experiment on day 39 after $\mathrm{T}$ cell transfer. The bars represent levels of cytokines of untreated rats and rats treated with RTL220 starting at the first or second onset as shown above. Cytokines were extracted from homogenized individual eyes with PBS and individually analyzed with a multiplex ELISA (Millipore) with antibodies specific for IL-1 $\beta$, TNF- $\alpha$, IL-6, IL-17, IFN$\gamma$, IL-2, IL-10 and IL-4 in triticale. T cells from rat individual spleens were stimulated with $20 \mu \mathrm{g} / \mathrm{ml}$ IRBP peptide for $48 \mathrm{~h}$ and then the supernatants were collected for cytokine measurements. Beadlyte multiplex cytokine detection kit (Millipore) was used to measure cytokines as described in the methods. The significance between the control and treatment group was determined using Student's t test $\left({ }^{*} \mathrm{p}<0.05\right)$ and is denoted by an asterisk. $\mathbf{h}$ Histology of RTL220-treated and control eyes. The eyes were collected on day 39 after $\mathrm{T}$ cell transfer and then fixed in formalin followed by paraffin embedding. Five-micrometer sections were prepared and stained with H\&E. Representative cross-sections of the rat retinas; arrows point at inflammatory cells. 


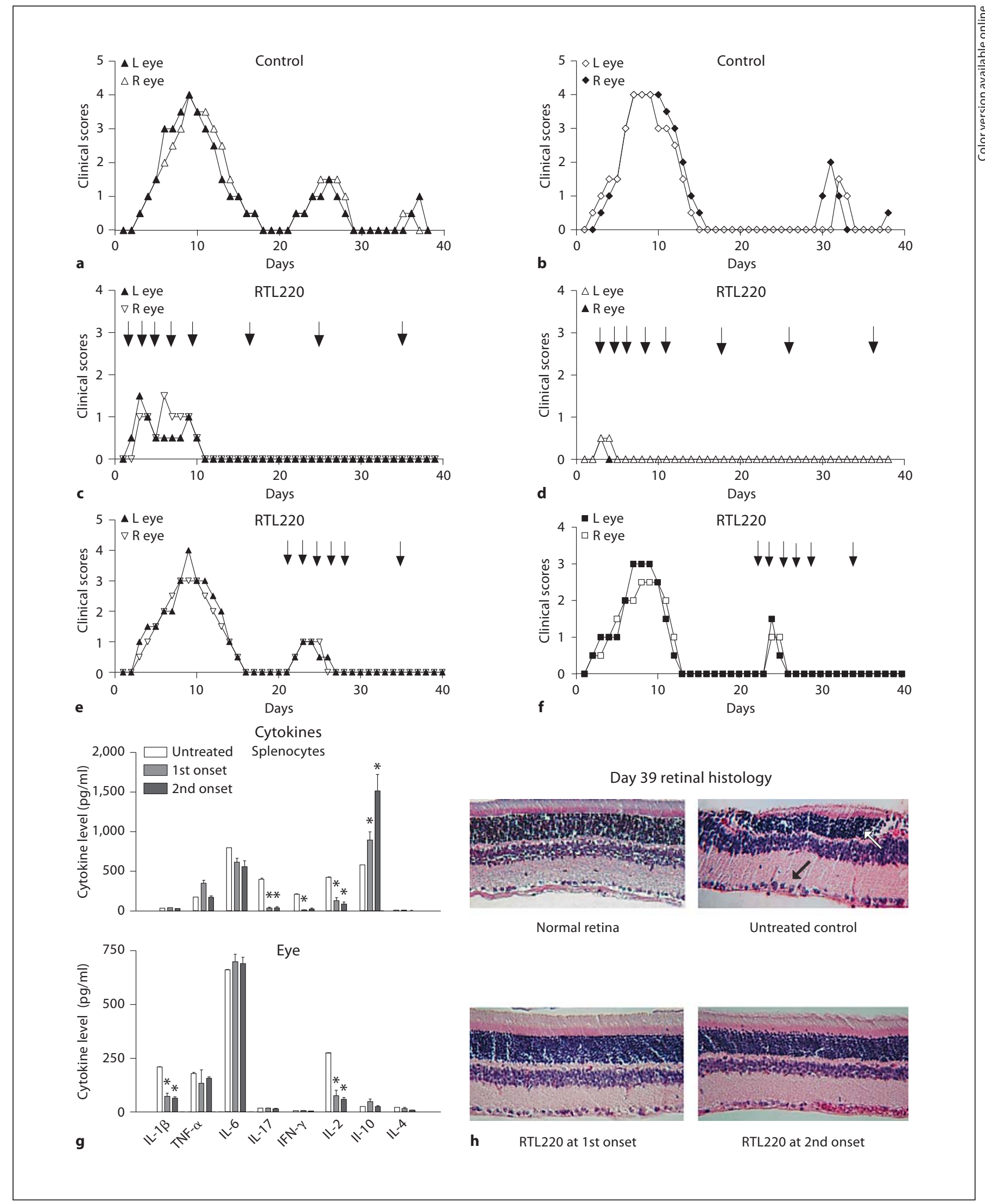

Treatment of Uveitis with RTL

Ophthalmic Res 2010;44:24-33 
$(n=6)-$ a score of 4 at the peak in control rats (fig.3a, b) compared to a score of $0.5-1$ at the peak in RTL220-treated rats (fig. 3c, d). When RTL220 was administrated at the second onset of inflammation, (fig. 3e, f; regimen 2) more than $50 \%$ reduction in severity of the second bout of inflammation (score 2-3) compared to control rats (score 1-2) was observed. The initial 5-dose treatment without weekly follow-up treatments had a diminished therapeutic effect with relapses (data not shown).

The CDI shows a significant difference between RTL220-treated $(2.625 \pm 2.5)$ and control groups (32.9 \pm 2.62 ) for the treatment started at the first attack of inflammation $(\mathrm{p}<0.0001, \mathrm{n}=6)$. For the second treatment regimen, the results also showed marked suppression of R-EAU, but the CDI did not reach statistical significance. The histology of R-EAU rats performed on eyes collected at the end of the experiments (39 days after immunization) also confirmed the clinical results (fig. $3 \mathrm{~h}$ ). The retina from the RTL220-treated rats showed a fully preserved morphology compared to that of the untreated rats, in which we found retinal weaving and local loss of photoreceptor cells, as well as signs of neovascularization in some rat retinas.

To further analyze the therapeutic effects of RTL220 we examined inflammatory cytokines and chemokines present in R-EAU in the eye and periphery, then compared it to the untreated rats. Splenocytes obtained from individual spleens of the different treatment groups were stimulated with the immunizing peptides for $48 \mathrm{~h}$ and supernatants were evaluated for cytokine secretion. Our results showed that both regimes of RTL220 treatments of EAU led to a significant reduction in proinflammatory cytokines secreted by spleen and eye cells (fig. 3g). The reduction in IL-17, IL- 2 and IFN- $\gamma$ was observed for splenic secretion, whereas only IL-2 and IL-1 $\beta$ were decreased in treated eyes. The systemic IL-10 levels were increased compared to the untreated controls, especially in the rats treated at the second onset. Other cytokines in the eyes were similar to the levels of those of the untreated controls, possibly due to collection of the samples at the end of the experiment.

The chemokines CCL2, CCL3 and CCL5 were upregulated at the onset of clinical symptoms, but the RTL220 treatment showed a significant suppression of the secretion of these chemokines in the periphery (fig. 4). The levels of CCL2 and CCL5 were reduced in the periphery and in the eye, which agrees with the lack of inflammatory cells in the eye. The level of CCL2 was suppressed in the spleen of treated animals, but the levels of CCL3 in the eye were measured very low, below $10 \mathrm{pg} / \mathrm{ml}$.

\section{Discussion}

The ultimate goal of new immunotherapies is to inhibit the ongoing disease process by modulating effector mechanisms. Our aim was to suppress ongoing inflammation with RTL220 and also protect the neuronal retina from retinal damage due to inflammation. Indeed, our studies showed that RTL220 suppressed clinical and histological signs of acute and recurrent IRBP-peptide-induced EAU. The prophylactic outcome was less successful in complete suppression, although the treatment prior to onset showed considerable protection of the retina from EAU. Importantly, treatment with RTL220 effectively abolished clinical and histological signs of relapses not only when delivered with the first onset of clinical disease but with later attacks of inflammations. The overall effect of this immunosuppression was a marked reduction in infiltrating cells in the eyes of the rats treated with RTL220 and decreased production of proinflammatory cytokines, such as IL-17 and IL-2, the major mediators of eye inflammation, and increased in anti-inflammatory IL-10 cytokine. The lack of inflammation in the eye may be due to an altered proinflammatory cytokine and chemokine expression in the periphery, thus preventing cell recruitment to the eye.

Visual loss is more common in posterior than anterior uveitis because of irreversible damage to the retina, which may be a consequence of the influx of the inflammatory cells and secretion of proinflammatory cytokines. Chronic uveitis involves ongoing priming and recruitment of new $\mathrm{T}$ cells into the effector pool and thus requires longterm interventional medical therapy. These studies offer a new treatment for recurrent uveitis using RTL immunotherapy targeting pathogenic $\mathrm{T}$ cells that in effect change their properties from pro- to anti-inflammatory.

RTL treatment acts in an antigen-specific manner, since RTLs without immunizing peptide (RTL101) or nonspecific peptide (RTL203) had no effect on the suppression of EAU. However, the antigen specificity of RTL raises a question as to whether treatment with single RTLs would be successful in uveitis patients where target antigens may be unknown. This is an ongoing project in our laboratory and future studies of treating animals immunized with the multiantigenic retinal homogenate will examine the effectiveness of such an approach. However, recently published studies on RTL treatment of EAE mice injected with spinal cord homogenate or combinations of 2 different peptides to induce disease showed that treatment with single RTLs could reverse EAE as long as targeted $\mathrm{T}$ cells were present in the periphery [26]. The 


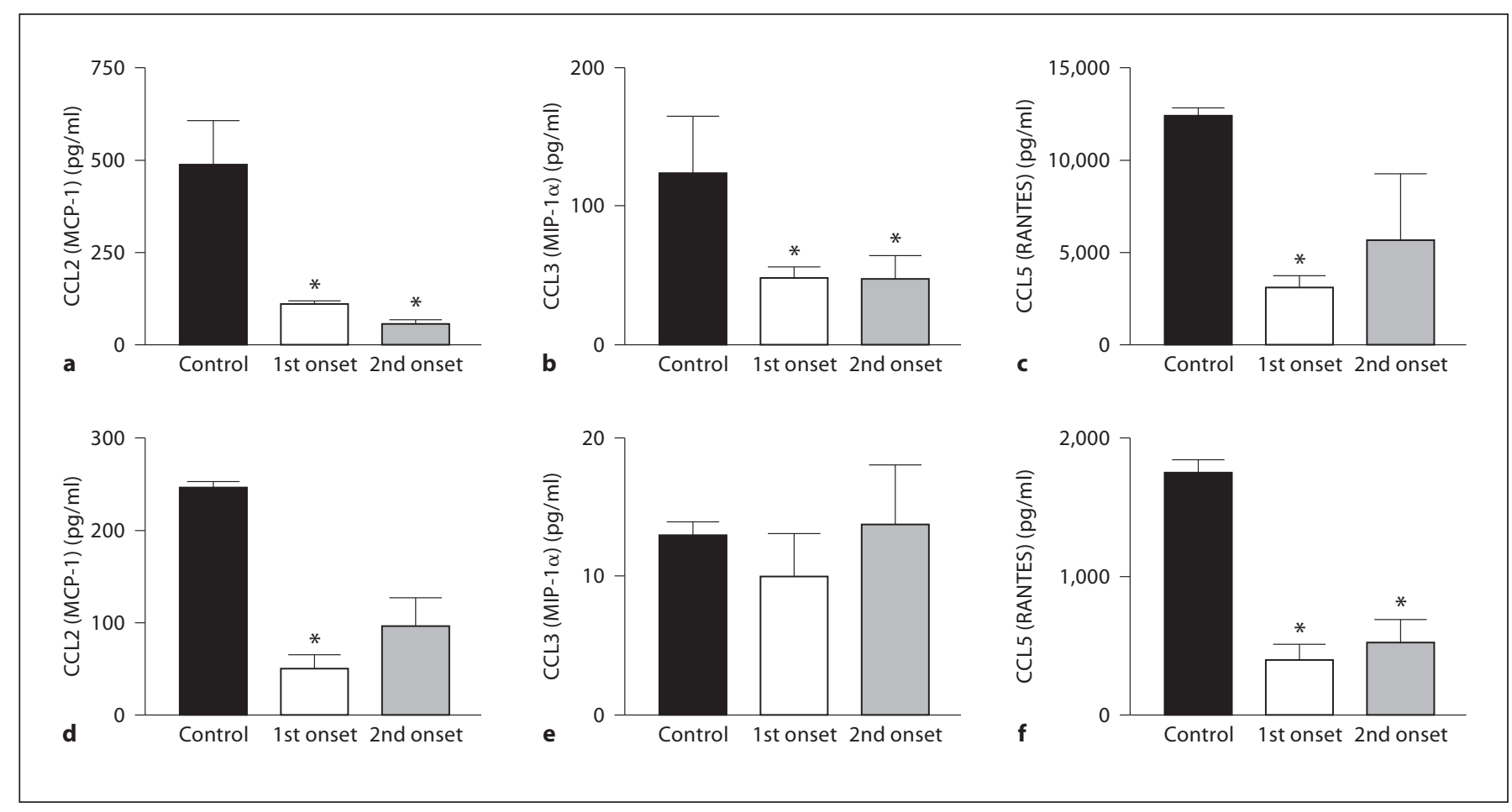

Fig. 4. Levels of systemic and local chemokines in R-EAU in RTL220-treated rats in comparison to the untreated rats. Eyes (df) and spleens $(\mathbf{a}-\mathbf{c})$ were collected at the end of the experiment on day 39 after $\mathrm{T}$ cell transfer. Untreated (first bar) rats, rats treated with RTL220 starting at the first onset (second bar) or starting at the second onset (third bar). Chemokines were extracted from homogenized eyes or from $48 \mathrm{~h}$ IRBP-stimulated T cell superna- tants for CCL2, CCL3 and CCL5 measurements and analyzed with a multiplex ELISA. A Beadlyte multiplex cytokine detection kit (Millipore) was used to measure cytokines as described in the methods and in figures 2 and 3 . The significance between the control and treatment group $(\mathrm{n}=4)$ was determined using Student's t test $\left({ }^{*} \mathrm{p}<0.05\right.$ denoted by an asterisk). authors believe that treatment with single RTLs can induce a cytokine switch in cognate $\mathrm{T}$ cells that inhibits both, the target and bystander $\mathrm{T}$ cells, providing new evidence for the potential applicability of RTL therapy in multiple sclerosis. Such a scenario could be possible in uveitis.

RTL treatment is a platform technology that we hope could be applicable for the treatment of many autoimmune diseases mediated by T cells. Thus far, RTLs have been successfully used in mouse experimental models of multiple sclerosis and rheumatoid arthritis, and these studies have revealed some details of an underlying mechanism of the therapeutic activity of RTLs. RTL engagement with TCR in the absence of CD4 binding was found to result in rapid TCR phosphorylation, calcium mobilization and reduced extracellular, signal-related kinase activity, as well as in a deviation from a Th1 to a Th0 cell phenotype based on cytokine production $[27,28]$. It has been suggested that elevated levels of IL10 induced in Th1 cells by RTLs have important regulatory implications for autoimmunity because IL-10 is known for its anti-inflammatory effects on Th1 cell and macrophage activation in EAE $[18,29]$. Besides the anti-inflammatory effect of IL-10 in EAE, it has been reported that intraocular expression of IL-10 by intravitreal injection of AAV2/2tetON-vIL-10 protected from S-Ag-induced EAU in Lewis rats with vIL-10 expressed over a long period of time [30]. Our experiments showed an increased secretion of IL-10 by splenocytes from RTL220-treated rats, suggesting that changes in cytokine expression may suppress the recruitment of inflammatory cells to the eye.

In general, RTL therapies in mice and rats inhibited the systemic production of pathogenic cytokines by the targeted specific $\mathrm{T}$ cells but also inhibited downstream, local recruitment and retention of inflammatory cells in the CNS as well as in the eye. In EAE studies using the C57BL/6 model, in which $\mathrm{IA}^{\mathrm{b}}$-restricted $\mathrm{T}$ cells specific for myelin oligodendrocyte glycoprotein peptide (MOG- 
35-45) are implicated in disease pathology, RTL551 (carrying covalently tethered MOG35-55 peptide) treatment of mice strongly and selectively reduced the secretion of IL-17 and TNF- $\alpha$, the latter of which was associated with the downregulation of chemokines and their receptors, and the inhibition of vascular cell adhesion molecule-1 and intercellular adhesion molecule-1 expression on endothelial cells [31]. IL-17 was also found to play a role in the pathogenesis of EAU, showing that systemic and local IL-17 response correlated with disease severity in EAU mice [32]. Targeting IL-17, even late in the disease process, ameliorated pathology, indicating an effector role for this cytokine in the pathogenesis of EAU [33]. Our results showed a considerably reduced systemic and local secretion of IL-17 after RTL220 treatment of acute and recurrent disease. Moreover, CCL2, CCL3 and CCL5 were suppressed in the eyes with EAU. It is widely accepted that synthesis and secretion of inflammatory chemokines play an important part in the pathogenesis of ocular inflammation $[34,35]$. Both CCL2 and CCL5 are associated with infiltrating inflammatory cells and are potent chemoattractants for $\mathrm{T}$ lymphocytes and macrophages, which are related to infiltrating cells observed in the posterior segment of eyes with EAU [34-36]. Thus, decreased chemokine levels by RTL220 may reflect a reduced number of recruited T lymphocytes and macrophages invading the retina during uveitis.

In summary, we demonstrated for the first time a successful treatment of acute and recurrent EAU. RTL220 effectively suppressed the recurrence of inflammation and reversed clinical and histological EAU by altering proinflammatory cytokine and chemokine expression, which possibly stopped the recruitment of pathogenic $\mathrm{T}$ cells into the eye. Such efficacy of RTLs in the therapy of anterior [19] and posterior uveitis (this study) strongly supports the possibility of clinical application of this novel class of peptide/MHC II drugs for patients with presumed autoimmune uveitis.

\section{Acknowledgement}

This work was supported by National Institutes of Health Grants EY017781 (G.A.), AI43960 (G.G.B.) and DK06881 (G.G.B.).

\section{References}

1 Maini R, O’Sullivan J, Reddy A, Watson S, Edelsten C: The risk of complications of uveitis in a district hospital cohort. Br J Ophthalmol 2004;88:512-517.

2 Tellier Z: Human immunoglobulins in intraocular inflammation. Ann NY Acad Sci 2007;1110:337-347.

-3 Yeh S, Faia L, Nussenblatt R: Advances in the diagnosis and immunotherapy for ocular inflammatory disease. Semin Immunopathol 2008;30:145-164

4 Adamus G, Chan CC: Experimental autoimmune uveitides: multiple antigens, diverse diseases. Int Rev Immunol 2002;21:209229.

5 Luger D, Caspi R: New perspectives on effector mechanisms in uveitis. Semin Immunopathol 2008;30:135-145.

-6 Agarwal RK, Caspi RR: Rodent models of experimental autoimmune uveitis. Methods Mol Med 2004;102:395-419.

7 Sun B, Sun SH, Chan CC, Wiggert B, Caspi RR: Autoimmunity to a pathogenic retinal antigen begins as a balanced cytokine response that polarizes towards type 1 in a disease-susceptible and towards type 2 in a disease-resistant genotype. Int Immunol 1999; 11:1307-1312.
8 Davey MP, Rosenbaum JT: The human leukocyte antigen complex and chronic ocular inflammatory disorders. Am J Ophthalmol 2000;129:235-243.

-9 Pennesi G, Caspi RR: Genetic control of susceptibility in clinical and experimental uveitis. Int Rev Immunol 2002;21:67-88.

10 Imrie FR, Dick AD: Biologics in the treatment of uveitis. Curr Opin Ophthalmol 2007;18:481-486.

11 Wildner G, Diedrichs-Möhring M, Thurau S: Rat models of autoimmune uveitis. Ophthalmic Res 2008;40:141-144.

12 Kezuka T, Sakai J, Yokoi H, Takeuchi M, Okada A, Taguchi O, Usui M, Mizuguchi J: Peptide-mediated suppression of experimental autoimmune uveoretinitis in mice: development of a peptide vaccine. Int Immunol 1996;8:1229-1235.

$\checkmark 13$ Sharma SD, Nag B, Su XM, Green D, Spack E, Clark BR, Sriram S: Antigen-specific therapy of experimental allergic encephalomyelitis by soluble class II major histocompatibility complex-peptide complexes. Proc Natl Acad Sci USA 1991;88:11465-11469.
14 Sasamoto Y, Kawano YI, Bouligny R, Wiggert B, Chader GJ, Gery I: Immunomodulation of experimental autoimmune uveoretinitis by intravenous injection of uveitogenic peptides. Invest Ophthalmol Vis Sci 1992;33: 2641-2649.

$\checkmark 15$ Burrows GG, Chang JW, Bachinger HP, Bourdette DN, Offner H, Vandenbark AA: Design, engineering and production of functional single-chain $\mathrm{T}$ cell receptor ligands. Protein Eng 1999;12:771-778.

16 Burrows GG: Systemic immunomodulation of autoimmune disease using MHC-derived recombinant TCR ligands. Curr Drug Targets Inflamm Allergy 2005;4:185.

17 Burrows GG, Bebo BF, Adlard KL, Vandenbark AA, Offner H: Two-domain MHC class II molecules form stable complexes with MBP-69-89 peptide that detect and inhibit rat encephalitogenic T cells and treat experimental autoimmune encephalomyelitis. J Immunol 1998;161:5987-5996.

18 Huan J, Subramanian S, Jones R, Rich C, Link J, Mooney J, Bourdette DN, Vandenbark AA, Burrows GG, Offner H: Monomeric recombinant TCR ligand reduces relapse rate and severity of experimental autoimmune encephalomyelitis in SJL/J mice through cytokine switch. J Immunol 2004; 172:4556-4566. 
19 Adamus G, Burrows GG, Vandenbark AA, Offner H: Treatment of autoimmune anterior uveitis with recombinant TCR ligands. Invest Ophthalmol Vis Sci 2006;47:25552561.

-20 Caspi RR, Chan CC, Wiggert B, Chader GJ: The mouse as a model of experimental autoimmune uveoretinitis (EAU). Curr Eye Res 1990;9:169-174.

21 Gery I, Michizuki M, Nussenblatt RB: Retinal specific antigens and immunopathogenic process they provoke; in Progress in Retinal Research. Oxford, Pergamon Press, 1986.

-22 Sanui H, Redmond TM, Kotake S, Wiggert B, Hu LH, Margalit H, Berzofsky JA, Chader GJ, Gery I: Identification of an immunodominant and highly immunopathogenic determinant in the retinal interphotoreceptor retinoid-binding protein (IRBP). J Exp Med 1989;169:1947-1960.

-23 Shao H, Lei S, Sun SL, Kaplan HJ, Sun D: Conversion of monophasic to recurrent autoimmune disease by autoreactive T cell subsets. J Immunol 2003;171:5624-5630.

>24 Caspi RR, Roberge FG, Chan CC, Wiggert B, Chader GJ, Rozenszajn LA, Lando Z, Nussenblatt RB: A new model of autoimmune disease: experimental autoimmune uveoretinitis induced in mice with two different retinal antigens. J Immunol 1988;140:14901495.

25 Adamus G, Manczak M, Machnicki M: Expression of CC-chemokines and their receptors in the eye in autoimmune anterior uveitis associated with EAE. Invest Ophthalmol Vis Sci 2001;42:2894-2903.
26 Sinha S, Subramanian S, Miller L, Proctor TM, Roberts C, Burrows GG, Vandenbark AA, Offner H: Cytokine switch and bystander suppression of autoimmune responses to multiple antigens in experimental autoimmune encephalomyelitis by a single recombinant T-cell receptor ligand. J Neurosci 2009;29:3816-3823

-27 Wang C, Mooney JL, Meza-Romero R, Chou YK, Huan J, Vandenbark AA, Offner H, Burrows GG: Recombinant TCR ligand induces early TCR signaling and a unique pattern of downstream activation. J Immunol 2003; 171:1934-1940.

28 Burrows GG, Adlard KL, Bebo BF Jr, Chang JW, Tenditnyy K, Vandenbark AA, Offner H: Regulation of encephalitogenic T cells with recombinant TCR ligands. J Immunol 2000; 164:6366-6371.

29 Burrows GG, Chou YK, Wang C, Chang JW Finn TP, Culbertson NE, Kim J, Bourdette DN, Lewinsohn DA, Lewinsohn DM, Ikeda M, Yoshioka T, Allen CN, Offner H, Vandenbark AA: Rudimentary TCR signaling triggers default IL-10 secretion by human Th1 cells. J Immunol 2001;167:4386-4395.

30 Smith JR, Verwaerde C, Rolling F, Naud MC, Delanoye A, Thillaye-Goldenberg B, Apparailly F, De Kozak Y: Tetracycline-inducible viral interleukin-10 intraocular gene transfer, using adeno-associated virus in experimental autoimmune uveoretinitis. Hum Gene Ther 2005;16:1037-1046.
31 Sinha S, Subramanian S, Proctor TM, Kaler LJ, Grafe M, Dahan R, Huan J, Vandenbark AA, Burrows GG, Offner H: A promising therapeutic approach for multiple sclerosis: recombinant T-cell receptor ligands modulate experimental autoimmune encephalomyelitis by reducing interleukin-17 production and inhibiting migration of encephalitogenic cells into the CNS. J Neurosci 2007;27:12531-12539.

32 Peng Y, Han G, Shao H, Wang Y, Kaplan HJ, Sun D: Characterization of IL-17+ interphotoreceptor retinoid-binding protein-specific $\mathrm{T}$ cells in experimental autoimmune uveitis. Invest Ophthalmol Vis Sci 2007;48:41534161.

33 Luger D, Silver PB, Tang J, Cua D, Chen Z, Iwakura Y, Bowman EP, Sgambellone NM, Chan CC, Caspi RR: Either a Th17 or a Th1 effector response can drive autoimmunity: conditions of disease induction affect dominant effector category. J Exp Med 2008;205: 799-810.

-34 Crane IJ, McKillop-Smith S, Wallace CA, Lamont GR, Forrester JV: Expression of the chemokines MIP-1 $\alpha$, MCP-1, and RANTES in experimental autoimmune uveitis. Invest Ophthalmol Vis Sci 2001;42:1547-1552.

35 Crane IJ, Xu H, Wallace C, Manivannan A, Mack M, Liversidge J, Marquez G, Sharp PF, Forrester JV: Involvement of CCR5 in the passage of Th1-type cells across the bloodretina barrier in experimental autoimmune uveitis. J Leukoc Biol 2006;79:435-443.

\36 Adamus G, Machnicki M, Amundson D, Adlard K, Offner H: Similar pattern of MCP1 expression in spinal cords and eyes of Lewis rats with EAE and associated anterior uveitis. J Neurosci Res 1997;50:531-538. 


\section{Erratum}

To the article by Adamus G, Karren LJ, Mooney J and Burrows GG, entitled 'A promising therapeutic approach for treatment of posterior uveitis: recombinant $\mathrm{T}$ cell receptor ligand protects Lewis rats from acute and recurrent experimental autoimmune uveitis' [Ophthalmic Res 2010;44:24-33, DOI: 10.1159/000281815], the following Disclosure Statement is to be added:

OHSU and Dr. Burrows have a significant financial interest in Artielle Immunotherapeutics Inc., a company that may have a commercial interest in the results of this research and technology. This potential conflict of interest has been reviewed and managed by OHSU. 